\title{
Access to alcohol and heart disease among patients in hospital: observational cohort study using differences in alcohol sales laws
}

\author{
Jonathan W Dukes, ${ }^{1}$ Thomas A Dewland, ${ }^{2}$ Eric Vittinghoff, ${ }^{3}$ Jeffrey E Olgin,, ${ }^{1}$ Mark J Pletcher, ${ }^{2}$ \\ Judith A Hahn, ${ }^{4}$ Rachel A Gladstone, ${ }^{1}$ Gregory M Marcus ${ }^{1}$
}

${ }^{1}$ Division of Cardiology,

Department of Medicine, University of California, 505

Parnassus Ave, M-1180B, Box

0124, San Francisco, CA

94143-0124, USA

${ }^{2}$ Knight Cardiovascular Institute, Oregon Health and Science University, Portland, OR, USA ${ }^{3}$ Department of Epidemiology and Biostatistics, University of California, San Francisco, CA USA

${ }^{4}$ Department of Medicine, University of California, San Francisco, CA, USA

Correspondence to:

G M Marcus

marcusg@medicine.ucsf.edu Additional material is published online only. To view please visit the journal online.

Cite this as: BMJ 2016;353:i2714 http://dx.doi.org/10.1136/bmj.i2714

Accepted: 20 April 2016

\section{ABSTRACT}

\section{OBJECTIVE}

To investigate the relation between alcohol

consumption and heart disease by using differences in county level alcohol sales laws as a natural experiment.

DESIGN

Observational cohort study using differences in alcohol sales laws.

\section{SETTING}

Hospital based healthcare encounters in Texas, USA. POPULATION

1106968 patients aged 21 or older who were residents of "wet" (no alcohol restrictions) and "dry" (complete prohibition of alcohol sales) counties and admitted to hospital between 2005 and 2010, identified using the Texas Inpatient Research Data File.

\section{OUTCOME MEASURES}

Prevalent and incident alcohol misuse and alcoholic liver disease were used for validation analyses. The main cardiovascular outcomes were atrial fibrillation, acute myocardial infarction, and congestive heart failure.

RESULTS

Residents of wet counties had a greater prevalence and incidence of alcohol misuse and alcoholic liver disease. After multivariable adjustment, wet county residents had a greater prevalence (odds ratio 1.05,

\section{WHAT IS ALREADY KNOWN ON THIS TOPIC}

The association between alcohol (the most commonly consumed drug in the world) and heart disease (the most common cause of death) is complex and often conflicting

The available evidence suggests that alcohol consumption may be associated with a higher risk of atrial fibrillation, a lower risk of myocardial infarction, and varying associations with heart failure

Previous observational studies have relied almost entirely on self reported alcohol use, resulting in the potential for substantial confounding regarding associated outcomes

\section{WHAT THIS STUDY ADDS}

Laws limiting alcohol sales have measurable public health effects, and increased access to alcohol is associated with more admissions for alcohol misuse and alcoholic liver disease

Increased access to alcohol is consistently associated with more atrial fibrillation, but fewer myocardial infarctions

Effects on atrial fibrillation are observed within only a few years after liberalization of alcohol sales laws
95\% confidence interval 1.01 to $1.09 ; \mathrm{P}=0.007$ ) and incidence (hazard ratio $1.07,1.01$ to $1.13 ; \mathrm{P}=0.014$ ) of atrial fibrillation, a lower prevalence (odds ratio 0.83 , 0.79 to $0.87 ; \mathrm{P}<0.001$ ) and incidence (hazard ratio $0.91,0.87$ to $0.99 ; \mathrm{P}=0.019)$ of myocardial infarction, and a lower prevalence (odds ratio $0.87,0.84$ to 0.90 ; $\mathrm{P}<0.001)$ of congestive heart failure. Conversion of counties from dry to wet resulted in statistically significantly higher rates of alcohol misuse, alcoholic liver disease, atrial fibrillation, and congestive heart failure, with no detectable difference in myocardial infarction.

\section{CONCLUSIONS}

Greater access to alcohol was associated with more atrial fibrillation and less myocardial infarction and congestive heart failure, although an increased risk of congestive heart failure was seen shortly after alcohol sales were liberalized.

\section{Introduction}

Alcohol is the most widely consumed drug in the United States. ${ }^{1}$ After the end of Prohibition, multiple states enacted laws permitting counties and local municipalities to place restrictions on the sale of alcohol. The most populous state to enact such laws was Texas, where "local option" laws have created a patchwork of counties where alcohol sales are unrestricted ("wet" counties), partially restricted, or completely prohibited ("dry" counties). The health effects of such laws have not been previously studied.

Heart disease remains the most common cause of death in the world. ${ }^{2}$ Alcohol may be an important determinant of heart disease, but the evidence is complex and often conflicting. Although the available evidence suggests that alcohol may increase the risk of atrial fibrillation $^{3-7}$ and reduce the risk of myocardial infarction, ${ }^{8-11}$ the data remain inconsistent. ${ }^{3-15}$ These results rely almost entirely on self reported alcohol use. Given the inaccuracies inherent to such ascertainment, ${ }^{16} 17$ misclassification of alcohol consumption may be an important problem that is challenging to overcome. In the absence of a controlled clinical trial, this primary predictor (alcohol consumption) is determined by the choice of each individual in all population based studies that have been described, setting the stage for substantial confounding that even the most comprehensive multivariable adjustment may be inadequate to overcome. As access to alcohol in residents of wet compared with dry counties is essentially assigned, we sought to use that assignment as a natural experiment. A particularly unique opportunity arises from counties that 
have converted from dry to wet, providing a comparison (the change in access to alcohol) within the same population that should minimize effects from both measured and unmeasured confounders. We therefore sought to investigate the relation between alcohol sales laws and hospital admissions for common cardiac diseases.

\section{Methods}

Information on the alcohol sales laws and local option election results for all counties in the state of Texas from 2005 and 2010 came from the Texas Alcoholic Beverage Commission. We also obtained all county-wide and municipal local option election records from 1937 to 2010 from the same source and reviewed them to validate a county's alcohol sales status. We defined a dry county as a county that prohibited all alcohol sales in all locations in the county throughout the follow-up time period and a wet county as one that had no prohibition on alcohol sales over the course of the follow-up period. We did two analyses. Firstly, we did a double cohort analysis wherein we compared cross sectional and longitudinal patient level outcomes among patients residing in wet versus dry counties, excluding patients residing in counties with partial restrictions on alcohol sales or where alcohol laws had changed during the study period. We used a double cohort design to longitudinally examine outcomes among two groups, each selected on the basis of the primary predictor (in this case, the presence versus absence of alcohol sales laws restrictions). Secondly, we did longitudinal analyses of a cohort of residents in counties that changed alcohol sales laws during the study period by comparing outcomes before and after the conversion.

We obtained county level median income by using the 2010 census data from the United States Census Bureau (http://quickfacts.census.gov). County hospital bed counts came from the Dartmouth Atlas of Healthcare (http://www.dartmouthatlas.org).

We used the Texas Inpatient Research Data File obtained from the Texas Department of State Health Services (Austin, TX) to identify all patients older than 21 years admitted to a hospital in the state of Texas between 1 January 2005 and 31 December 2009 $(\mathrm{n}=4832460)$. We linked individual databases for each calendar year by using an encrypted linkage variable specific to each patient, allowing for longitudinal follow-up of an individual over time. We excluded patients with missing admission date, county of residence, age, sex, race, or ethnicity data or residence outside of the state of Texas $(\mathrm{n}=195741)$. Patients were entered into the study cohort at the time of their first admission and followed until 31 December 2009 for diagnosis of the outcome of interest or censoring at the time of inpatient death.

The admitting institution recorded age, sex, race, ethnicity, and county of residence at each healthcare encounter. We defined patients' county of residence at the time of their first hospital admission. Up to 24 ICD-9 (international classification of diseases, 9th revision) codes were provided for each encounter to identify outcomes of interest and relevant covariates (supplementary table A). Dichotomous medical comorbidities were accumulated at each healthcare encounter and carried forward over time. We did a proof of concept analysis using alcohol misuse and alcoholic liver disease as outcomes. The primary heart disease outcomes of interest were atrial fibrillation, acute myocardial infarction, and congestive heart failure. As postoperative atrial fibrillation may have a different underlying mechanism than atrial fibrillation occurring outside of the acute surgical setting, we did not include diagnoses of atrial fibrillation in the analysis if the patient had undergone cardiothoracic surgery during the same hospital admission or within the previous 30 days. For the analyses of prevalent diseases, we used only ICD-9 codes present at the time of the first hospital admission. In analyses of incident disease, we excluded patients with the outcome of interest at the time of their first hospital admission.

\section{Patient involvement}

This research used de-identified patient data. No patients were involved in setting the research question or the outcome measures, nor were they involved in developing plans for the design or implementation of the study. No patients were asked to advise on interpretation or writing up of results. There are no plans to disseminate the results of the research to study participants or the relevant patient community.

\section{Statistical analysis}

Continuous variables with a normal distribution are presented as means and standard deviations and were compared using Student's $t$ test. Non-normally distributed continuous variables are presented as medians with interquartile ranges and were compared using Kruskal-Wallis tests. Associations between categorical variables were assessed using $\chi^{2}$ tests.

We used logistic regression for multivariable analyses of dichotomous outcomes. We estimated differences in the cumulative incidence of each outcome by using a Fine Gray competing risks model, ${ }^{18}$ with in-hospital death treated as a competing risk. We used Cox proportional hazards models to investigate incident outcomes after multivariable adjustment. Patients who had more than one outcome, such as atrial fibrillation, then myocardial infarction, then congestive heart failure, would remain in the model until the outcome of interest occurred; the relevant comorbidities (such as atrial fibrillation or myocardial infarction before congestive heart failure) were included accordingly as time updated covariates. We tested hazard proportionality assumptions graphically using smoothed scaled Schoenfeld estimates, which showed no violation of the proportional hazard assumption. All analyses were controlled for potential confounders identified a priori that were available in the administrative dataset and selected on the basis of biological plausibility and convention determined by previous literature.

Owing to significant differences in the baseline covariates between the wet and dry counties, we also did propensity adjusted and sensitivity analyses. We used propensity score methods to estimate adjusted relative 
hazards for the association of baseline county wet-dry status with alcohol misuse, alcoholic liver disease, atrial fibrillation, acute myocardial infarction, and congestive heart failure by using the multivariable adjusted Cox models. The Cox models were then adjusted for the tenth of the propensity score. Selection of covariates for integration into these propensity scores was based on published recommendations to include all available potential confounders identified a priori while avoiding inclusion of mediators or colliders and erring on the side of inclusion when potential confounders were known to be particularly important to the outcome (as opposed to only the predictor) relevant to the analysis of interest. ${ }^{1920}$ The specific covariates included for each outcome are listed in supplementary table B. We evaluated the models used to estimate the propensity scores by plotting the observed versus expected percentages living in wet counties (supplementary figure). Visual inspection of these plots showed acceptable calibration. Given known differences in cardiovascular outcomes by race, ethnicity, and sex, ${ }^{721}$ we did sensitivity analyses by restricting the cohorts to non-Hispanic white men.

To compare rates of admission for heart disease before and after changes in alcohol sales laws, we used logistic models to compare disease prevalence in the two periods among admitted residents of the counties that changed wet/dry status. We also did county level ecologic analyses, in which we calculated population based admission rates by using the population of the each county. We then used Poisson models to estimate and compare adjusted admission rates.

We used Stata 12 for all analyses. We considered a two tailed $\mathrm{P}$ value less than 0.05 to be statistically significant.

\section{Results}

Twenty nine counties were identified as dry, 47 counties were wet, and seven counties changed from dry to wet. After applying exclusion criteria, we identified 4662123 patients aged 21 or older who were admitted to hospital in the state of Texas between 1 January 2005 and 31 December 2009. The dry counties had prohibited alcohol sales since Prohibition, and the most recent year that any of the wet counties had been dry was 1959.

\section{Patient level analyses in wet versus dry counties}

Among 1046759 wet county residents with at least one hospital admission, a total of 2388700 admissions occurred. In dry counties, 60209 residents were admitted to hospital, resulting in 139192 admissions. Table 1 shows the baseline characteristics of these patients. Table 2 shows the crude (unadjusted) prevalence and incidence of each outcome, as well as the same summary statistics restricted to non-Hispanic white men, in wet and dry counties.

\section{Differences in alcohol misuse and alcoholic liver disease as proof of concept}

After adjustment for age, race, ethnicity, and sex, the prevalence of alcohol misuse was significantly greater among residents of wet counties than in dry counties (fig 1). Also after multivariable adjustment, we found a $36 \%$ greater risk of incident alcohol misuse among admitted residents of wet counties than in dry counties (hazard ratio 1.36, 1.23 to $1.50 ; \mathrm{P}<0.001$ ). After adjustment for the same covariates, prevalent alcoholic liver disease was also more common in admitted wet county residents (fig 1). Similarly, incident alcoholic liver disease was higher among patients residing in wet counties, with an adjusted hazard ratio of 1.81 (1.51 to 2.16; $\mathrm{P}<0.001)$.

\section{Cardiovascular diagnoses}

Atrial fibrillation was significantly more prevalent among wet county residents after adjustment for age,

\begin{tabular}{|c|c|c|c|}
\hline Variable & Dry county $(n=60209)$ & Wet county $(n=1046759)$ & $P$ value \\
\hline Median (interquartile range) age, years & $60(42-74)$ & $50(32-69)$ & $<0.001$ \\
\hline Female sex & $35549(59)$ & $687465(66)$ & $<0.001$ \\
\hline \multicolumn{4}{|l|}{ Race: } \\
\hline White & $49435(82)$ & $581327(56)$ & $<0.001$ \\
\hline Black & $5050(8)$ & $57999(6)$ & \\
\hline Asian/Pacific Islander & $84(0.1)$ & $12277(1)$ & \\
\hline Native American & $452(0.8)$ & $15272(1)$ & \\
\hline Other & $5077(8)$ & $378402(36)$ & \\
\hline Hispanic ethnicity & $6345(11)$ & $554239(53)$ & $<0.001$ \\
\hline Obesity & $4414(7)$ & $76719(7)$ & 0.99 \\
\hline Hypertension & $27206(45)$ & $386725(37)$ & $<0.001$ \\
\hline Coronary artery disease & $12508(21)$ & $144579(14)$ & $<0.001$ \\
\hline Diabetes & $12174(20)$ & $221406(21)$ & $<0.001$ \\
\hline Chronic kidney disease & $1940(3)$ & $39843(4)$ & $<0.001$ \\
\hline Valvular heart disease & $2379(4)$ & $29126(3)$ & $<0.001$ \\
\hline Pulmonary disease & 9619 (16) & $103603(10)$ & $<0.001$ \\
\hline Congestive heart failure & $6560(11)$ & $78847(8)$ & $<0.001$ \\
\hline Acute myocardial infarction & $2392(4)$ & $27395(3)$ & $<0.001$ \\
\hline Atrial fibrillation & $4543(8)$ & $52002(5)$ & $<0.001$ \\
\hline
\end{tabular}




\begin{tabular}{|c|c|c|c|c|c|}
\hline \multirow[b]{2}{*}{ Outcome } & \multicolumn{2}{|c|}{ Prevalence (\%) } & \multirow{2}{*}{$\begin{array}{l}\text { Median } \\
\text { (interquartile range) } \\
\text { years of follow-up }\end{array}$} & \multicolumn{2}{|c|}{$\begin{array}{l}\text { Incidence (per } 1000 \\
\text { person years) }\end{array}$} \\
\hline & Wet & Dry & & Wet & Dry \\
\hline Alcohol misuse & 2.6 & 2.5 & $2.9(1.5-4.1)$ & 3.6 & 2.5 \\
\hline Sensitivity* & 5.0 & 4.4 & $2.9(1.4-4.1)$ & 4.7 & 3.5 \\
\hline Alcoholic liver disease & 0.7 & 0.4 & $2.9(1.5-4.1)$ & 1.4 & 0.8 \\
\hline Sensitivity* & 0.9 & 0.6 & $2.9(1.4-4.1)$ & 2.2 & 1.3 \\
\hline Atrial fibrillation & 4.9 & 7.5 & $2.9(1.5-4.1)$ & 7.4 & 10.0 \\
\hline Sensitivity $^{\star}$ & 10.7 & 10.0 & $2.8(1.4-4.1)$ & 12.8 & 13.9 \\
\hline Acute myocardial infarction & 2.6 & 4.0 & $2.9(1.5-4.1)$ & 5.4 & 6.7 \\
\hline Sensitivity* & 4.8 & 6.3 & $2.9(1.4-4.1)$ & 7.4 & 8.7 \\
\hline Congestive heart failure & 7.1 & 10.4 & $2.9(1.5-4.0)$ & 12.5 & 16.0 \\
\hline Sensitivity* & 10.5 & 12.2 & $2.8(1.4-4.0)$ & 16.9 & 18.5 \\
\hline
\end{tabular}

race, ethnicity, sex, diabetes, hypertension, obesity, tobacco abuse, congestive heart failure, coronary artery disease, lung disease, and valvular disease (fig 1). Similarly, wet counties residents had a significantly greater adjusted incidence of atrial fibrillation than did patients residing in dry counties (hazard ratio 1.07, 1.01 to $1.13 ; \mathrm{P}=0.014)$.

After adjustment for age, race, ethnicity, sex, diabetes, hypertension, obesity, and tobacco use, wet county resident status was associated with a significantly lower odds of acute myocardial infarction on the first hospital admission observed (fig 1). Residents of wet counties also had a significantly lower risk of incident acute myocardial infarction (adjusted hazard ratio $0.91,0.87$ to $0.99 ; \mathrm{P}=0.019$ ). After multivariable adjustment for age, race, ethnicity, sex, diabetes, hypertension, obesity, tobacco use, acute myocardial infarction, and coronary artery disease, wet county residents had a significantly lower odds of prevalent congestive heart failure (fig 1). After adjustment for the same potential cofounders, wet

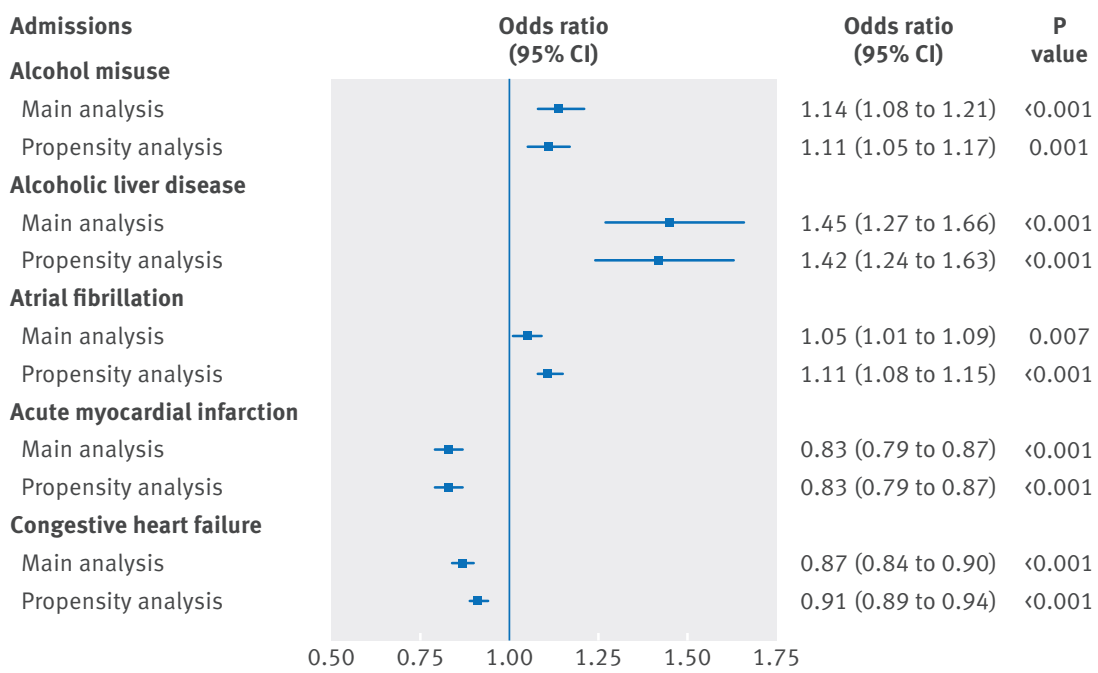

Fig 1 Association between wet county residence and prevalent hospital admissions. Squares represent main and propensity score adjusted analyses odds ratios for prevalent alcohol misuse, alcoholic liver disease, atrial fibrillation, acute myocardial infarction, and congestive heart failure in wet county residents compared with dry county residents (see text for list of covariates included in each model) country residents had a decreased risk of incident congestive heart failure that did not meet statistical significance (adjusted hazard ratio 0.98, 0.94 to 1.03; $\mathrm{P}=0.431)$.

\section{Propensity analyses}

The propensity adjusted analyses showed no meaningful differences in prevalent disease compared with the conventional multivariable adjusted analyses (fig 1). In the incidence analyses, only one meaningful difference was observed: with propensity adjustment, the association of wet county status with incident congestive heart failure maintained the same point estimate hazard ratio but became statistically significant (0.96, 0.91 to $1.00 ; \mathrm{P}=0.045$ ). Figure 2 shows the propensity adjusted cumulative incidence curves.

\section{Conversion of county status}

During the five years of observation, no counties converted from wet to dry. However, seven dry counties passed countywide referendums lifting restrictions on alcohol sales. The total number of hospital admissions before and after the referendums in those counties were 43559 and 42734 over a median duration of 15 (interquartile range 7-22) months before and 15 (8-25) months after the law change. The post-conversion interval (from dry to wet county status) was associated with a greater odds of alcohol misuse (odds ratio $1.31,1.19$ to $1.43 ; \mathrm{P}<0.001)$, alcoholic liver disease $(1.61,1.35$ to $1.91 ; \mathrm{P}<0.001)$, atrial fibrillation (1.07, 1.03 to $1.12 ; \mathrm{P}=0.001)$, and congestive heart failure $(1.07,1.04$ to $1.11 ; \mathrm{P}<0.001)$ among patients admitted to hospital; we found no difference in acute myocardial infarction (odds ratio 0.99, 0.91 to 1.07; $\mathrm{P}=0.746$ ). In an ecological analysis using the population of the counties as the denominator, we observed a higher incidence of alcohol misuse, alcoholic liver disease, atrial fibrillation, and congestive heart failure admissions per 1000 person years after the lifting of alcohol sale restrictions, whereas myocardial infarction showed no meaningful change (fig 3). Note that the incidence differences shown reflect the difference between the before and after referendum rates for a given outcome. 

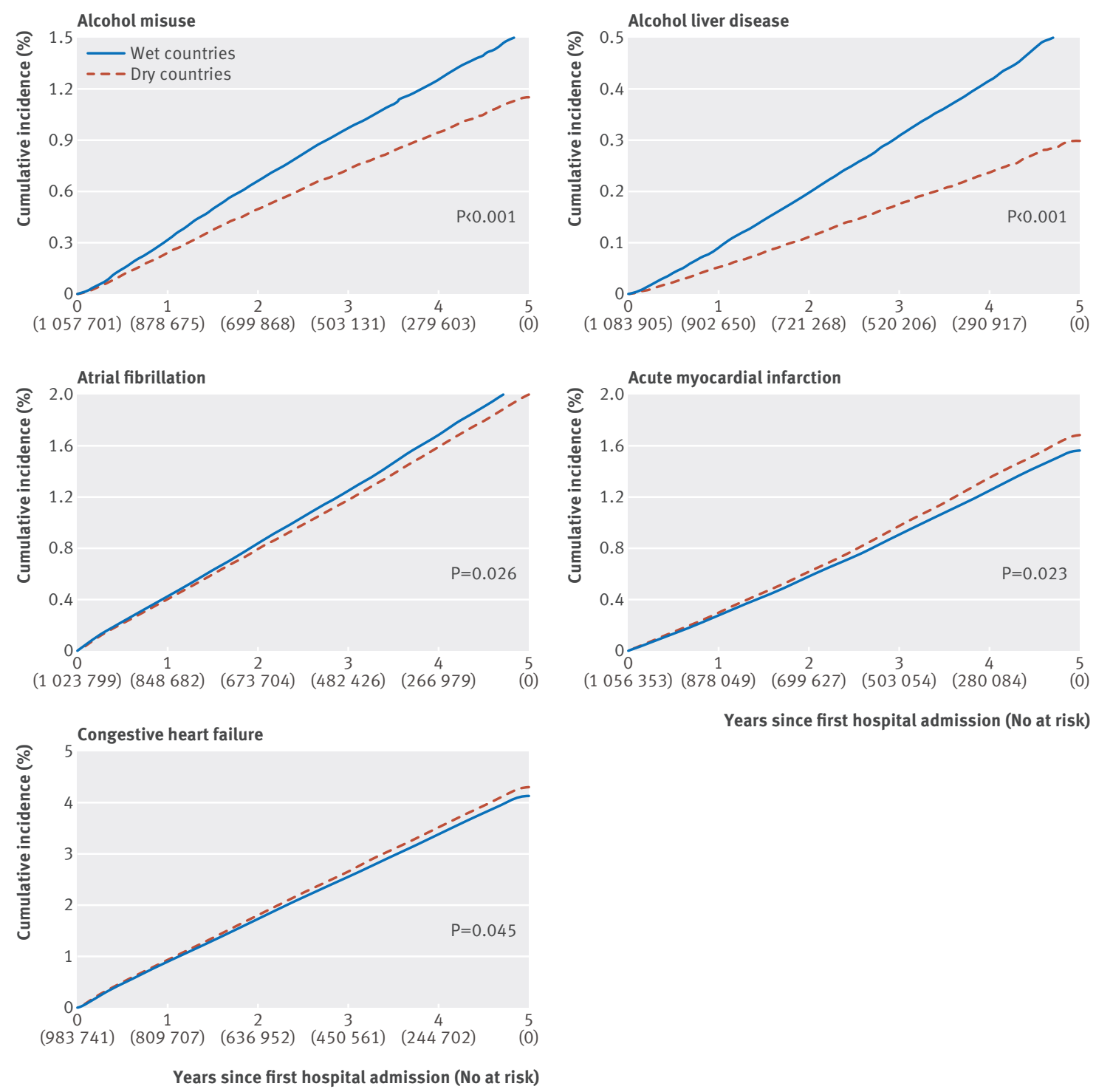

Years since first hospital admission (No at risk)

Fig 2 | Propensity score adjusted cumulative incidence for alcohol misuse, alcoholic liver disease, atrial fibrillation, acute myocardial infarction, and congestive heart failure admissions in dry and wet counties. Stratified by dry and wet counties in Texas between 1 January 2005 and 31 December 2010. Numbers in parentheses represent number at risk in study population. See text for the covariates included in each model

\section{Discussion}

Among all patients admitted to hospital in Texas from 2005 to 2010, those residing in a county without alcohol sales restrictions had a higher prevalence and incidence of alcohol misuse, alcoholic liver disease, and atrial fibrillation; a lower prevalence and incidence of acute myocardial infarction; and a lower prevalence and incidence of congestive heart failure. Over the same five years, conversion of counties from restrictive to open alcohol laws resulted in increased rates of alcohol misuse, alcoholic liver disease, atrial fibrillation, and congestive heart failure.

\section{Comparison with other studies}

Previous studies examining the associations between alcohol and heart disease have been limited by two major factors. Firstly, they have generally relied on self report, which is not necessarily reliable. ${ }^{1617}$
Secondly, alcohol consumption among research participants is primarily based on each individual's discretion. This makes extricating the associations between alcohol consumption and other related behaviors, decisions, and exposures that may serve as important confounders difficult if not impossible. Our approach eliminates concerns about the accuracy of self reported alcohol consumption. This allows our alternative research approach to complement and add clarity to the previous survey based studies. Most importantly, the before versus after intra-county analyses provide outcome data in the same population with and without restriction of alcohol sales, delivering a particularly potent approach to minimize confounding. The validity of the use of dry and wet county status as a marker for alcohol use was shown by our alcohol misuse and alcoholic liver disease analyses. 


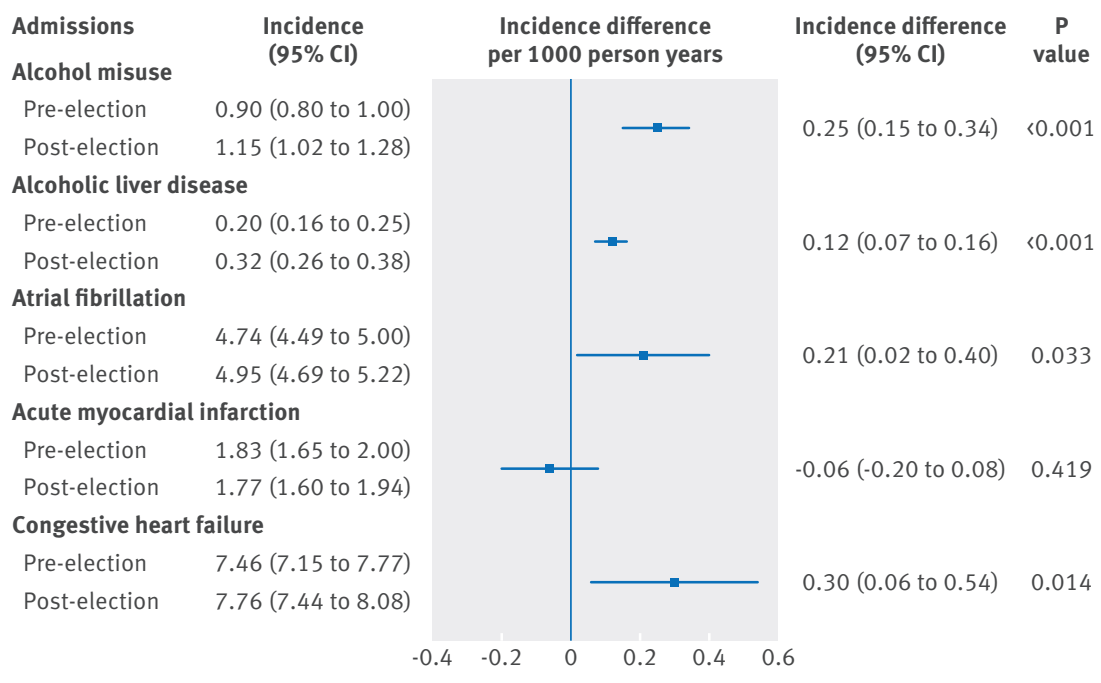

Fig 3 | Change in incidence of alcoholic liver disease, alcohol misuse, atrial fibrillation, acute myocardial infarction, and congestive heart failure admissions before and after conversion from dry to wet county status. Squares represent change in incident rates for each type of hospital admission in seven counties that converted from dry to wet. Denominators for incident rates were determined using 2010 US census data. Incident difference is difference between incident rates before and after referendum for given outcome. Error bars indicate $95 \%$ confidence intervals

Although most population based studies have suggested that increased alcohol consumption is associated with a higher risk of incident atrial fibrillation, 562223 not all reports have identified a significant association. ${ }^{347}$ In this study, wet county residents had more atrial fibrillation, whether analyzed as a prevalent or incident outcome. Atrial fibrillation was the only outcome that showed divergent results between the unadjusted numbers and the adjusted results in the intra-county analyses. Such results were in fact expected owing to two important considerations: firstly, dry county residents were older and more often male and non-Hispanic white, all particularly important risk factors for atrial fibrillation; ${ }^{721}$ secondly, owing to the inverse relations between county status and myocardial infarction and congestive heart failure, some "negative confounding" (sometimes termed "suppression") was likely, which, once accounted for, uncovered wet county status as a risk factor for atrial fibrillation. Propensity based analyses also showed that atrial fibrillation was more common in wet counties. As this database tracked patients on their county of residence, and not by the location of the admitting hospital, these findings are unlikely to be explained by rural versus urban hospital referral patterns. These findings are further supported by our more robust intra-county before and after election analysis, which showed an increase in admissions for atrial fibrillation after the liberalization of drinking laws.

Although previous data generally favor a protective effect of alcohol on the risk of myocardial infarction, ${ }^{10} 11$ results from conventional epidemiologic studies have been conflicting. ${ }^{11-1324-26}$ Previous studies have used per capita alcohol consumption and population based outcomes to understand the relation between alcohol and cardiovascular health. ${ }^{24-27}$ Interestingly, the most consistent message is that more per capita alcohol consumption is associated with an increased mortality among patients with ischemic heart disease. ${ }^{25-27}$ However, none of these studies focused on incident myocardial infarction, heart failure, or atrial fibrillation. In our study, patients residing in wet counties had a lower risk of myocardial infarction. In contrast, no significant changes in admissions for myocardial infarction were observed after a change in alcohol sales laws within the same counties. Although this may suggest that some residual confounding in the wet versus dry county analyses remains, it may be that the chronic beneficial effects of alcohol had inadequate time to confer any demonstrable protection against myocardial infarction with changes in alcohol access over a relatively short timeframe. Most of the beneficial effects on coronary artery disease are thought to be chronic, including influencing pathways related to cholesterol transport, insulin sensitivity, abdominal obesity, and oxidative stress. ${ }^{28}$

The relation between heart failure and alcohol use has been one of the more complex associations to elucidate. On the one hand, alcohol is known to cause cardiomyopathy. ${ }^{2930}$ On the other hand, some studies have suggested that alcohol may have a protective effect when consumed in moderation. ${ }^{1415}$ Both atrial fibrillation and myocardial infarction can lead to heart failure, ${ }^{31} 32$ with myocardial infarction likely being much the more important of the two. As with myocardial infarction, wet county residents had significantly less prevalent congestive heart failure. In addition, the propensity based analysis showed a decreased incidence of congestive heart failure in wet county residents. After referendums to liberalize alcohol sales in the intra-county analyses, incidence of congestive heart failure increased. Once again, although we cannot exclude residual confounding in the between county analyses as an explanation for these disparate results, it may be that the acute effects of alcohol (known to result in negative inotropy ${ }^{3334}$ ) trump the beneficial chronic effects (such as via reduction in myocardial infarction) during this switch in access to alcohol over only one to two years. In contrast, the alcohol laws were stable for decades preceding the study timeframe of the between county analyses, which may have resulted in lower rates of myocardial infarction (through chronic protective effects against coronary artery disease) and thus lower overall rates of congestive heart failure in chronically wet versus chronically dry counties.

The discordant results, showing more atrial fibrillation but less myocardial infarction and congestive heart failure in wet compared with dry counties, are particularly remarkable given that these outcomes generally share all of the same risk factors and that each can lead to the other. Specifically, atrial fibrillation predicts myocardial infarction and congestive heart failure, ${ }^{31-37}$ just as myocardial infarction predicts atrial fibrillation 73839 and congestive heart failure predicts atrial fibrillation. ${ }^{731}$ Therefore, given these potential "downstream" influences, these discordant results may suggest that 
the direct effects of alcohol are more potent than the point estimates reported.

\section{Strengths and limitations of study}

Although this is the largest study of alcohol use and heart disease and the first to use differences in alcohol sales laws as a predictor of disease, we must acknowledge several limitations. As alcohol laws in the state of Texas limit only the sale of alcohol in a municipality and not its transport or consumption, misclassification of the exposure in the form of alcohol consumption in dry counties is possible. This misclassification could have attenuated the observed effects. We cannot exclude the possibility that patterns of practice in one country differed (such as having a lower threshold to admit to hospital for cardiovascular disease), but our discordant findings between the atrial fibrillation and the myocardial infarction and congestive heart failure results strongly suggest that no systematic difference in referral patterns for hospital admission existed. The Texas Inpatient Research Data File is an administrative database that is derived from physicians' admission and discharge coding. Although we were not able to validate physicians' coding for an individual patient (all data are de-identified), our predictor (access to alcohol) did not rely on physicians' coding and differences in alcohol misuse and alcoholic liver disease validated our assumptions. Most acute myocardial infarctions that come to medical attention likely result in a hospital admission, but we would have failed to capture out of hospital fatal myocardial infarctions and episodes of atrial fibrillation and congestive heart failure treated in emergency departments or outpatient clinics. As mentioned throughout the manuscript, residual confounding as an explanation for our results is an important consideration. Some potentially important covariates, such as individual alcohol exposure, county level per capita alcohol use, dietary habits, physical activity, and disease severity, were not available in this dataset. However, most of our results persisted after both multivariable adjustment and propensity analyses, as well as in the robust change analyses within the same counties. Finally, we acknowledge that these observational data are insufficient to prove any causal relations.

Dry counties were on average less wealthy (supplementary table C). Although differences in socioeconomic status might explain some of the results observed, individual level measures of income or education were not available in this dataset. We did not adjust for county level income in the between county analyses owing to collinearity with the primary predictor. Importantly, it would seem to be unlikely that differences in socioeconomic status could explain discordant results on the relation between alcohol and atrial fibrillation compared with the myocardial infarction and congestive heart failure outcomes (that is, more atrial fibrillation in wet counties, yet less myocardial infarction and congestive heart failure), and our previous publication using similar data from California and geographic location as a surrogate of socioeconomic status failed to show an association between location and atrial fibrillation after adjustment for race. ${ }^{21}$ Finally, this once again likely points to the superior reliability of the within county analyses, in which such factors would be expected to remain constant in a given county.

Dry counties also on average had fewer hospital beds. However, the location of the participants was based on their county of residence rather than where they sought medical care, and access to inpatient facilities would be determined primarily by insurance status and referral patterns of primary care physicians rather than county of residence. We also cannot take proximity of a hospital across county lines into account. Once again, it would seem unlikely that access to hospitals explained our discordant results (as more access in general would be expected to be associated with more of each diagnosis rather than more of some and less of others). Finally, the number of hospital beds in a particular county should not influence the within county analyses. Taken together, however, clear differences exist in the characteristics of dry versus wet counties, and we cannot exclude the possibility that some unmeasured confounder(s) apart from the alcohol access laws may explain the between county results. This is in part why our validation analyses, showing more alcohol misuse and more alcoholic liver disease in wet than dry counties, may be especially important in showing true differences in alcohol consumption between counties.

\section{Conclusions and policy implications}

We have shown, for the first time, that laws limiting alcohol sales have measurable public health effects. Increased access to alcohol was consistently associated with more atrial fibrillation and less myocardial infarction. Given that this investigation of the most commonly consumed drug (alcohol) and the leading cause of death (heart disease) leverages a natural experiment to minimize confounding, we believe that our results have broadly applicable health implications relevant to people with and at risk for various types of cardiovascular disease. More broadly, this research shows that public laws limiting access to a commonly consumed substance have important health effects that may be both beneficial and harmful.

Contributors: All authors were involved in the study design, data analysis, and revision of the manuscript. JWD and GMM wrote the manuscript. All authors read and approved the final manuscript. JWD is the guarantor

Funding: Research reported in this publication was supported by the National Institute on Alcohol Abuse and Alcoholism of the National Institutes of Health under award number R01AA022222 (GMM). The funders of the study had no role in the study design, data collection, data analysis, data interpretation, writing of the report, or the decision to submit the article for publication.

Competing interests: All authors have completed the ICMJE uniform disclosure form at www.icmje.org/coi_disclosure.pdf and declare: no support from any organization for the submitted work other than that described above; GMM has received research support from Medtronic and Pfizer and is a consultant and equity holder in InCarda; no other relationships or activities that could appear to have influenced the submitted work

Ethical approval: Use of the Texas Inpatient Research Data File was approved by the Texas Department of State Health Services Institutional Review Board and certified by the University of California, San Francisco Committee on Human Research.

Data sharing: No additional data available. 
Transparency: The lead author affirms that the manuscript is an honest, accurate, and transparent account of the study being reported; that no important aspects of the study have been omitted; and that any discrepancies from the study as planned (and, if relevant, registered) have been explained.

This is an Open Access article distributed in accordance with the Creative Commons Attribution Non Commercial (CC BY-NC 3.0) license, which permits others to distribute, remix, adapt, build upon this work non-commercially, and license their derivative works on different terms, provided the original work is properly cited and the use is noncommercial. See: http://creativecommons.org/licenses/by-nc/3.0/.

1 Maxwell JC. Update: comparison of drug use in Australia and the United States as seen in the 2001 National Household Surveys. Drug Alcohol Rev 2003:22:347-57. doi:10.1080/0959523031000154490.

2 Mendis S, Puska P, Norrving B, eds. Global Atlas on cardiovascular disease prevention and control. World Health Organization, 2011.

3 Krahn AD, Manfreda J, Tate RB, Mathewson FA, Cuddy TE. The natural history of atrial fibrillation: incidence, risk factors, and prognosis in the Manitoba Follow-Up Study. Am J Med 1995;98:476-84. doi:10.1016/S0002-9343(99)80348-9.

4 Stewart S, Hart CL, Hole DJ, McMurray IJ. Population prevalence, incidence, and predictors of atrial fibrillation in the Renfrew/Paisley study. Heart 2001;86:516-21. doi:10.1136/heart.86.5.516.

5 Mukamal KJ, Tolstrup IS, Friberg J, Jensen G, Grønbaek M. Alcoho consumption and risk of atrial fibrillation in men and women: the Copenhagen City Heart Study. Circulation 2005;112:1736-42. doi:10.1161/CIRCULATIONAHA.105.547844.

6 Kodama S, Saito K, Tanaka S, et al. Alcohol consumption and risk of atrial fibrillation: a meta-analysis. J Am Coll Cardiol 2011;57:427-36. doi:10.1016/j.jacc.2010.08.641

7 Benjamin EJ, Levy D, Vaziri SM, D’Agostino RB, Belanger Al, Wolf PA Independent risk factors for atrial fibrillation in a population-based cohort. The Framingham Heart Study. JAMA 1994;271:840-4. doi:10.1001/jama.1994.03510350050036.

8 O'Keefe JH, Bybee KA, Lavie CJ. Alcohol and cardiovascular health: the razor-sharp double-edged sword. J Am Coll Cardiol 2007;50:1009-14. doi:10.1016/j.jacc.2007.04.089

9 Hammar N, Romelsjö A, Alfredsson L. Alcohol consumption, drinking pattern and acute myocardial infarction. A case referent study based on the Swedish Twin Register. I Intern Med 1997;241:125-31. doi:10.1046/j.1365-2796.1997.92106000.x

10 Ferrari P, Licaj I, Muller DC, et al. Lifetime alcohol use and overall and cause-specific mortality in the European Prospective Investigation into Cancer and nutrition (EPIC) study. BMJ Open 2014;4:e005245. doi:10.1136/bmjopen-2014-005245.

11 Leong DP, Smyth A, Teo KK, et al. INTERHEART Investigators. Patterns of alcohol consumption and myocardial infarction risk: observations from 52 countries in the INTERHEART case-control study. Circulation 2014:130:390-8. doi:10.1161/CIRCULATIONAHA.113.007627.

12 Mukamal KJ, Conigrave KM, Mittleman MA, et al. Roles of drinking pattern and type of alcohol consumed in coronary heart disease in men. N Engl/ Med 2003:348:109-18. doi:10.1056/NEIMoa022095.

13 Holmes MV, Dale CE, Zuccolo L, et al. InterAct Consortium. Association between alcohol and cardiovascular disease: Mendelian randomisation analysis based on individual participant data. BMJ 2014;349:g4164. doi:10.1136/bmj.g4164.

14 Walsh CR, Larson MG, Evans JC, et al. Alcohol consumption and risk for congestive heart failure in the Framingham Heart Study. Ann Intern Med 2002:136:181-91. doi:10.7326/0003-4819-136-3-200202050-00005.

15 Bryson CL, Mukamal KJ, Mittleman MA, et al. The association of alcohol consumption and incident heart failure: the Cardiovascular Health Study. J Am Coll Cardiol 2006;48:305-11. doi:10.1016/j. jacc.2006.02.066.

16 Heeb J-L, Gmel G. Measuring alcohol consumption: a comparison of graduated frequency, quantity frequency, and weekly recall diary methods in a general population survey. Addict Behav 2005;30:40313. doi:10.1016/j.addbeh.2004.04.022.

17 Del Boca FK, Darkes J. The validity of self-reports of alcohol consumption: state of the science and challenges for research. Addiction 2003;98(Suppl 2):1-12. doi:10.1046/j.1359-6357.2003.00586.X

18 Fine JP, Gray RJ. A proportional hazards model for the subdistribution of a competing risk. J Am Stat Assoc 1999;94:496-509. doi:10.1080/0 1621459.1999.10474144.
19 Sauer BC, Brookhart MA, Roy J, VanderWeele T. A review of covariate selection for non-experimental comparative effectiveness research. Pharmacoepidemiol Drug Saf 2013:22:1139-45. doi:10.1002/ pds.3506.

20 Heinze G, Jüni P. An overview of the objectives of and the approaches to propensity score analyses. Eur Heart J 2011;32:1704-8. doi:10.1093/eurheartj/ehr031.

21 Dewland TA, Olgin JE, Vittinghoff E, Marcus GM. Incident atrial fibrillation among Asians, Hispanics, blacks, and whites. Circulation 2013:128:2470-7. doi:10.1161/CIRCULATIONAHA.113.002449.

22 Frost L, Vestergaard P. Alcohol and risk of atrial fibrillation or flutter: a cohort study. Arch Intern Med 2004;164:1993-8. doi:10.1001/ archinte.164.18.1993.

23 Djoussé L, Levy D, Benjamin E), et al. Long-term alcohol consumption and the risk of atrial fibrillation in the Framingham Study. Am J Cardio 2004:93:710-3. doi:10.1016/i.amjcard.2003.12.004

24 Hemström O. Per capita alcohol consumption and ischaemic heart disease mortality. Addiction 2001;96(Suppl 1):S93-112. doi:10.1080/09652140020021206.

25 Kerr WC, Karriker-Jaffe K, Subbaraman M, et al. Per capita alcohol consumption and ischemic heart disease mortality in a panel of US states from 1950 to 2002. Addiction 2011;106:313-22. doi:10.1111/j.1360-0443.2010.03195.x.

26 Ramstedt M. Fluctuations in male ischaemic heart disease mortality in Russia 1959-1998: assessing the importance of alcohol. Drug Alcohol Rev 2009;28:390-5. doi:10.1111/j.1465-3362.2009.00059.x.

27 Ramstedt M. Is alcohol good or bad for Canadian hearts? A time-series analysis of the link between alcohol consumption and IHD mortality. Drug Alcohol Rev 2006;25:315-20. doi:10.1080/09595230600741057.

28 Krenz M, Korthuis RJ. Moderate ethanol ingestion and cardiovascular protection: from epidemiologic associations to cellular mechanisms. J Mol Cell Cardiol 2012;52:93-104. doi:10.1016/i.yjmcc.2011.10.011.

29 Komajda M, Richard IL, Bouhour JB, et al. Dilated cardiomyopathy an the level of alcohol consumption: a planned multicentre case-control study. Eur Heart J1986;7:512-9.

30 McKenna Cl Codd MB, McCann HA, Sugrue DD Alcohol consumption and idiopathic dilated cardiomyopathy: a case control study. Am Heart/1998;135:833-7. doi:10.1016/S0002-8703(98)70042-0.

31 Wang TJ, Larson MG, Levy D, et al. Temporal relations of atrial fibrillation and congestive heart failure and their joint influence on mortality: the Framingham Heart Study. Circulation 2003;107:2920-5. doi:10.1161/01.CIR.0000072767.89944.6E.

32 He J, Ogden LG, Bazzano LA, Vupputuri S, Loria C, Whelton PK. Risk factors for congestive heart failure in US men and women: NHANES I epidemiologic follow-up study. Arch Intern Med 2001;161:996-1002. doi:10.1001/archinte.161.7.996

33 Regan RI, Koroxenidis G, Moschos CB, Oldewurtel HA, Lehan PH, Hellems HK. The acute metabolic and hemodynamic responses of the left ventricle to ethanol. J Clin Invest 1966;45:270-80. doi:10.1172/JC1105340.

34 Kelbaek H, Giørup T, Brynjolf I, Christensen NJ, Godtfredsen J. Acute effects of alcohol on left ventricular function in healthy subjects at rest and during upright exercise. Am J Cardiol 1985;55:164-7. doi:10.1016/0002-9149(85)90320-0.

35 Soliman EZ, Safford MM, Muntner P, et al. Atrial fibrillation and the risk of myocardial infarction. JAMA Intern Med 2014;174:107-14. doi:10.1001/jamainternmed.2013.11912.

36 Shinbane IS, Wood MA, Jensen DN, Ellenbogen KA, Fitzpatrick AP, Scheinman MM. Tachycardia-induced cardiomyopathy: a review of animal models and clinical studies. J Am Coll Cardiol 1997;29:709-15. doi:10.1016/S0735-1097(96)00592-X

37 Grogan M, Smith HC, Gersh BJ, Wood DL. Left ventricular dysfunction due to atrial fibrillation in patients initially believed to have idiopathic dilated cardiomyopathy. Am J Cardiol 1992;69:1570-3. doi:10.1016/0002-9149(92)90705-4

38 Psaty BM, Manolio TA, Kuller LH, et al. Incidence of and risk factors for atrial fibrillation in older adults. Circulation 1997;96:2455-61. doi:10.1161/01.CIR.96.7.2455.

39 Jabre P, Jouven X, Adnet F, et al. Atrial fibrillation and death after myocardial infarction: a community study. Circulation 2011;123:2094100. doi:10.1161/CIRCULATIONAHA.110.990192.

\section{Supplementary materials}

